\title{
On High Brightness Temperature of Pulsar Giant Pulses
}

\author{
Victor M. Kontorovich ${ }^{1,2}$ \\ 1. Decametric Wave Department, Institute of Radio Astronomy NAS of Ukraine, Kharkov 61002, Ukraine \\ 2. Radio Physics, Mechanics and Mathematics Departments, V. N. Karazin National University, Kharkov 61022, Ukraine
}

\begin{abstract}
A wide range of events observed at the giant pulses (high energy density, observed localization of giant pulses GPs relative to the average pulse, fine structure of GPs with duration up to some nanoseconds, observed circular polarization of GPs, correlation between the GP phase and the phase of the hard pulsar emission X-ray and gamma) can be explained from the viewpoint that the internal polar gap is a cavity-resonator stimulated by discharges and radiating through the breaks in the magnetosphere. The new results in this field [the electromagnetic (em) waves generation in the gap in the process of longitudinal acceleration in the electric field vanishing on the star surface, high frequency break in the spectrum as a result of switching off this generation, formation in this process a power-low spectrum with a high frequency (hf) break, the possibility determination of pulsar magnetic field by the hf break position, the difference between main pulse and inter pulse mechanism generation, quantization of em tornado rotation in the gap and appearance of the bands in the inter pulse spectrum, influence the high energy density in the gap on pair generation and position of the dead line in pulsars] are added in the intermediate epilogue.
\end{abstract}

Key words: Pulsars, giant pulses, inner gap, cavity-resonator, spark rotation, electromagnetic tornado.

\section{Nomenclature}

$\begin{array}{ll}\text { GP: } & \text { Giant pulse } \\ \text { hf: } & \text { High frequency } \\ \text { em: } & \text { Electromagnetic }\end{array}$

\section{Greek letters}

$\begin{array}{ll}\rho_{G J}: & \text { Density charge of Goldreich-Julian } \\ \Sigma_{P C}: & \text { Area of the polar cap }\end{array}$

\section{Introduction}

Giant pulses (GPs), sporadically observed in a small number of pulsars ${ }^{1}$, are a riddle which has not yet been solved [1-5]. GP is characterized by enormous flux density [6], extremely small pulse duration (down to a few nanoseconds) [7], presence of circular polarization of both directions [8], power distribution by energies [9], mainly location in the narrow window with respect to the average pulse

Corresponding author: Victor M. Kontorovich, doctor of sci, professor, research fields: theoretical physics, plasma physics, and astrophysics. E-mail: vkont1001@yahoo.com; vkont@rian.kharkov.ua.

${ }^{1} \mathrm{Crab} \quad(\mathrm{B} 0531+21), \quad \mathrm{B} 1937+21, \quad \mathrm{~B} 1821-24, \quad \mathrm{~B} 0540-69$, B1112+50, B1957+20, J0218+4232, J1823-3021A, B0031-07, $\mathrm{J} 1752+2359, \mathrm{~B} 0656+146,[31,33-35,37-42]$. position [10] and correlation between the GP phase and the phase of the hard pulsar emission (X-ray and $\gamma$-ray) [11, 12]. All these features fundamentally distinguish GPs from ordinary pulses ${ }^{2}$. Nevertheless, GP seems to be "a frequent, but rarely observed phenomenon inherent in all pulsars" [10]. A number of pulsars ${ }^{3}$ emits anomalously intensive pulses [13] which by their properties seemingly do not differ from GPs. Trying to explain GPs by plasma mechanisms in magnetosphere in which different variants of two-stream instability are realized [14] needs considering strongly nonlinear effects such as modulation instability $[15,16]$, Zakharov plasma wave collapse (the more popular) [8], reconnection of the magnetic field lines $[17,18]$, induced scattering in narrow beams $[5,19]$.

When explaining the GPs in these works, we have seen that the pulsar is regarded as a "plasmic generator", a "device", in which the radiation processes are governed by different (nonlinear)

\footnotetext{
${ }^{2}$ See also the works [43-48].

${ }^{3} \mathrm{~B} 0809+74$, B $0823+26$, B0834+06, B0943+10, B0950+08, B1133+16.
} 
processes in the magnetosphere plasma.

In the Ref. [10], it is indicated that GPs are characterized by the extremely high energy density of order $10^{15} \mathrm{erg} / \mathrm{cm}^{3}$, which appears as a key moment and forms the basis of this study.

It should be emphasized, and this is the main subject-matter of this work that in the similar terms, the pulsar can be thought of as a "vacuum device", in which the magnetospheric plasma acts as the walls, limiting the cavity and the waveguides as well the breaks in the magnetosphere, through which the radiation of GPs is transmitted. The radiation itself arises at discharges in the inner gap.

Thus we proceed from the idea that the pulsar internal polar gap [20], where the particle acceleration processes occur in the longitudinal electric field [21], is a cavity-resonator (with respect to the low-frequency radiation [22, 23]). In the Ref. [22], the idea of resonator was illustrated by analogy with the resonator "earth-ionosphere" excited by the lightning discharges: "We live in a resonant cavity bounded below by the earth and above by the ionosphere. Lightning strikes excite low-frequency electromagnetic Schumann resonances in this cavity, as predicted by Schumann (1952), with a fundamental frequency of about 8 Hz." Such behaviour should also occur above the magnetic poles of pulsars due to the well-defined boundaries ${ }^{4}$ "found there" [22]. The modes of the cylindrical resonator were used in Ref. [22] to describe such feature as a "carusel" structure of the pulsar micro pulses, and, as seen from the quotation above, the analogy with Schuman resonance in the spherical "earth-ionosphere" resonator (e.g., the book [24] of Bliokh and his collaborators) was discussed.

In the Ref. [23], another aspect of the problem is brought to the foreground: the stationary random process of discharges on the polar cap surface and, as a result, formation of a stable average shape of the pulse. That was demonstrated with the aid of the toy dice model. The idea of the resonator and waveguides was also formulated and then used in the context of pulsar radio and hard radiation [25] from the polar gap. Owing to this idea, the radiation emanates from the gap through the waveguides in the neighborhood of the magnetic axis and through the slots [26] at the open field line borders. The radiation also leaks out through the magnetosphere plasma. The correlation between radio and gamma radiation, arising due to inverse Compton scattering of accelerated electrons at a powerful low-frequency radiation, can be an indirect confirmation of the powerful oscillations in the gap [25]. GP may be another and more evident manifestation of the strong oscillations in the gap.

In this work, we distinguish ideas between two approaches that we propose. One of them is to consider the passage of powerful quasi-stationary oscillations in the cavity through the random breaks in the magnetosphere. Under the assumption, the inner gap serves as a resonator (Section 2 and Appendix B). With the other approach, we examine (to explain the GP microstructure) the direct radiation of the individual discharge by-passing the plasma through the slot and the waveguide. This allows explain both the nanosecond pulse duration (due to relativistic aberration in the fast-rotating pulsars) and the observed circular polarization of GPs (Section 3 and Appendix C). The last approach does not use the concept of the polar gap as a cavity. Similar notions and views may turn out to be useful in analyzing the properties of ordinary pulses, their substructure, subpulse drift, etc. However, these attempts will doubtless be restricted to the lack of developed concepts of the "random" magnetosphere of open field lines and the most probable routes of the radiation transmission through such a medium. These questions are briefly discussed in Appendix A in the application to the random magnetosphere which is a stationary one on average only.

\footnotetext{
${ }^{4}$ See also discussion in Appendix A
} 


\section{Description of the Model}

In our opinion, GP can represent a direct emanation radiation from the gap (Fig. 1) through the breaks in the magnetosphere.

The particles accelerated in the gap undergo Compton losses, which exceed the curvature radiation losses under sufficient density of oscillation energy [25]. The energy received from the longitudinal electric field and, in the long run, from star rotation, is emanated in the form of gamma quanta (which generate the electron-positron plasma), and in the form of radio emission as in common models [27, 28].

The energy density $U$ in the gap can be estimated according to the law of electromagnetic field energy conservation in the cavity (Appendix B) stimulated by external discharge currents in view of the radiation losses (the irradiation through the waveguide). Under the stationary conditions, the square averages of these terms (the averages over space and time) have to be equal to each other. To estimate the scalar product of the current density and the electric field we use the Cauchy inequality. The energy density is determined as the square of the average electric field. We find the upper limit of energy density from the condition that the average current through the gap should be equal to the Goldreich-Julian current, which results in the following [25]:

$$
U \leq 4 \pi(1+\mu)^{2}\left(\Sigma_{P C} / \Sigma_{w}\right)^{2}\left(h \cdot \rho_{G J}\right)^{2}
$$

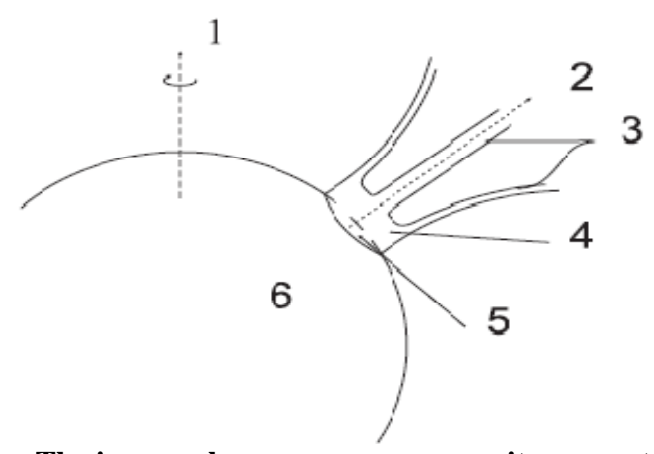

Fig. 1 The inner polar gap serves as a cavity-resonator for radio band electromagnetic oscillations excited by discharges in the gap. The radiation goes out through the wave-guides and percolates through the magnetosphere plasma. (1): rotation axis; (2): magnetic axis; (3): waveguides; (4): polar gap; (5): polar cap; (6): neutron star. where, $\Sigma_{P C}$ is the area of the polar cap, $\Sigma_{W}$ is the area of the waveguide cross-section, $\rho_{G J}$ is the density charge of Goldreich-Julian, $h$ is the average height of the gap, $\mu$ is the part of the resonator locked modes that cannot go out through the waveguides. The appropriate parameter estimation leads to the energy densities comparable with those observed at GPs: $U \leq 10^{16} \mathrm{erg} / \mathrm{s}$. The same condition may be transformed into the energy density restriction from below if we express it in the terms of radiation intensity $I_{R}$ :

$$
8 \pi U \geq\left(I_{R} / c \rho_{G J} h \cdot \Sigma_{p c}\right)^{2}
$$

which allows one to estimate $U \geq 10^{12} \mathrm{erg} / \mathrm{s}$. One more estimate of the energy density $U$ in the gap may be made by using the momentum conservation law. The very high level of the energy density in the gap means that the pressure of radiation in it has to be very high, too. It follows that the form of the boundary of the cavity with plasma must be sufficiently sharp. The boundary condition of the momentum density flux continuity gives $^{5}$ for $U$ :

$$
U \leq \kappa n_{G J} m c^{2} \Gamma_{\mathrm{sec}}^{2}
$$

where, $\quad n_{G J}=\rho_{G J} / e$ is the Goldreich-Julian concentration, $\kappa \approx 10^{3}$ the multiplicity factor, $\Gamma_{\sec }$ the Lorentz-factor of the secondary $e^{ \pm}$-plasma above the inner gap. Here we assume that the contribution of gamma-ray quanta in the flux is of the same order or smaller. It implies that the position of the control section in the plasma must be at a distance of some gamma quanta mean free pass above the boundary. The estimation $\Gamma_{\text {sec }} \approx 10^{3}-10^{4}$ gives us $U \leq 10^{14}-10^{15}$ $\mathrm{erg} / \mathrm{cm}^{3}$, the same as the previous ones on the rough scale. The more accurate estimation requires the solution of difficult problems of the plasma boundary structure and of the transmission (reflection) of the powerful electromagnetic waves through such a boundary. This problem is not yet solved. (In that case

${ }^{5} \mathrm{Cf}$. with the non-relativistic expression for momentum density flux $P+m n V^{2}, P$ is the pressure, $n$ is the particle concentration, $m$ is the mass of the particle. 
the linear approach to the problem is not applicable at all.)

Thus, we assume that a great amount of energy may actually be concentrated in the polar gap and the gap may be regarded as a cavity resonator. Some additional arguments in favor of this will be mentioned in the text below. We will discuss below the implications of these assumptions relative to the GPs and will confirm a consistency (noncontradictory) and efficiency of such a hypothesis.

The energy radiated by the GPs is determined by the oscillation energy density in the gap and parameters of the break in the magnetosphere plasma. The energy emanated in the pulse duration is proportional to the volume of the break $S \cdot \Delta z$, where, $\Delta z$ is its height proportional to radiation time $\Delta t$.

\section{Discussion}

For the distribution of the oscillation energy density by frequencies $U(v)$, we take the same power law with the index $\alpha_{R}>1$ as for the observed radio frequency radiation ${ }^{6}$

$$
\begin{gathered}
U(v)=U \cdot \frac{\left(\alpha_{R}-1\right)}{v_{\min }}\left(\frac{v_{\min }}{v}\right)^{\alpha_{R}} \\
U=\int_{v_{\min }}^{v_{2}} \mathrm{~d} v U(v)
\end{gathered}
$$

here, $v_{\min } \approx c / \lambda_{\max }$ is determined by the most long-wave modes, and $v_{2}$ is the maximal possible frequency of oscillations in the cavity corresponding to the high-frequency edge of the pulsar radio spectrum. The link between the break area $S$ and received flux density $F(v)$ on the given frequency has a form:

$$
U(v) \cdot c \cdot S=D^{2} \cdot \Delta \Omega \cdot F(v)
$$

here, $D$ is the distance from the pulsar, $\Delta \Omega$ is the spatial angle interval in which the radiation emanates. The azimuth angular distance $\Delta \varphi$ is passed during the

\footnotetext{
${ }^{6}$ Pulsar in the given model emanates GPs in the frequency interval $v_{1}<v<v_{2}$, where $v_{1} \approx c / \sqrt{ } S$ is determined by the conditions of transmission through the break (waveguide) of the given cross-section $S, v_{\min }<v<v_{1}$ is the interval of the locked modes.
}

time $\Delta t=P \cdot \Delta \varphi / 2 \pi$, where, $P$ is the period. Thus, the spatial angle $\Delta \Omega=\Delta \theta \cdot \Delta \varphi$ correlates with the time of radiation $\Delta t$ as $\Delta \Omega \approx 2 \pi \cdot \Delta \theta \cdot \Delta t / P$. The break area $S$ also requires the time $\Delta t$ for passing through. Taking into consideration $S<<\pi R_{s}^{2}, S=\pi R_{s}^{2} \Delta \Omega_{s}$ where, $R_{s}$ is the effective radius on which the break is realized and $\Delta \Omega_{s}$ is the corresponding spatial angle, we see that the length of the pulse $\Delta t$ falls out from our relations. If $\Delta \theta_{s} \approx \Delta \theta$ then we have [29]:

$$
\pi R_{s}^{2}=D^{2} F(v) /(U(v) \cdot c)
$$

or

$$
\pi R_{s}^{2}=\frac{D^{2} \cdot F(v) \cdot v_{\min }}{\left(\alpha_{R}-1\right) \cdot U \cdot c} \cdot\left(\frac{v}{v_{\text {min }}}\right)^{\alpha_{R}}
$$

The average GP profile is attached to the definite phase of the polar cap $\theta$. Hence the average form of the cavity arch $h(\theta) \approx R_{s}-R *$. Probably, near the magnetic axis this description is not bad. Yet, near the slot, it is necessary to take into consideration its curvature along with the other complicating factors [30].

Now we can make the independent rough estimate [29] of the energy density $U$ (from the top). Assume (only for this estimation!) that it makes the same contribution to each spectral interval of the observable density flux. If we make a sufficiently rough model to be considered in each frequency window $\mathrm{U}=$ constant, then for the spectral index $\alpha=3$, the area of the break falls out [29] from formulas:

$$
F(v)=(\alpha-1) S^{(3-\alpha) / 2} U \lambda^{\alpha} /\left(D^{2} \Delta \Omega\right)
$$

here, $D$ is the distance to the pulsar, $F(v)$ is the flux at a frequency $v, \lambda$ is the wavelength, $\Delta \Omega$ is the solid angle of radiation. Since $\alpha \approx 3$ corresponds to the spectrum of the Crab pulsar, we are able to evaluate $U$ from the observational data for Crab: $U=$ $D^{2} \cdot \Delta \Omega \cdot \lambda^{-3} \cdot F(v) / 2, \alpha=3$ (for this estimation only!). This gives us $U \leq 10^{17} \mathrm{erg} / \mathrm{cm}^{3}$, which is in good correlation with other estimates (the text above and Appendix B). 


\subsection{GP Short Duration}

An extremely short giant pulse duration of several nanoseconds allows us to suggest that the pulses arise in the inner gap [12] in the process of primary electron acceleration [13] to the gamma-factors of order of $10^{7}$. Indeed, relativistic aberration ${ }^{7}$ in the primary electron beam reduces the cone of radiation down up to the values $\delta \varphi \times 10^{-7}$, and in this case, the rotation with periods $P \approx 2 \pi 10^{-2} \mathrm{~s}$ leads to the nanosecond pulse duration $\delta t \approx \delta \varphi \cdot P / 2 \pi \times 10^{-9} \mathrm{~s}$. This explanation is also consistent with the fact that giant pulses are observed in the rapidly rotating pulsars only. Thus, we have to deal with the emission of an individual discharge in the inner gap [28].

\subsection{GP Phase}

The observed localization of the GP phase can be associated with radiation through the waveguides. In pulsar, B1112+50 GPs are located in the center of the average pulse [31]. We reckon that this localization may correspond to the radiation through the "waveguide" near the magnetic axis of the pulsar. If the GP phase corresponds to the "edge" of the average pulse, then it most likely corresponds to the radiation through the slot. The edge can be either retarded against the average profile $[9,32](\mathrm{B} 1937+21)$, or advanced [33] (J1823-3021A). This corresponds to trailing or leading edges of the slots in the section of the telescope diagram. The fine structure of the GPs may reflect the discreteness of the discharges [28] visible through the breaks.

From this point of view, the correlation between the GP phase and the phase of the hard pulsar emission (X-ray and $\gamma$-ray) $[1,11,12]$ becomes truly evident: radiation arises in the same process of particle acceleration and goes out through the same waveguides.

Localization of GPs (near the waveguides and near the slots) signifies that the magnetosphere of the open field lines is not transparent, on average, to radiation,

${ }^{7}$ See also the Ref. [49] except for these places of GP localization. This statement also supports the idea that the inner polar gap is a cavity resonator. ${ }^{8}$

\subsection{Circular Polarization and Electromagnetic Tornado}

The observed circular polarization $[8,32]$ is naturally explained by the peculiarities of the discharge in the inner gap. Coulomb charge repulsion in the discharge bunch furnishes the radial electric field orthogonal to the magnetic one. Owing to the drift in the crossed fields, it causes the discharge jet rotation around its axis (Appendix $\mathrm{C}$ ) and, accordingly, the circular polarization of generated electromagnetic waves. In fact, both accidental and regular deviations from the ideal axisymmetric form of discharges results in the rotational electric field around the discharge axes and the related circular polarization of radiation. Owing to the drift, the discharge channel turns into a peculiar vortex resembling the well-known tornado. However, contrary to the hydrodynamic nature of the usual tornado, the inner gap tornado is of purely electromagnetic origin.

From the equation $\operatorname{div} E=4 \pi \rho$ for the spatial charge $\rho$ of the form:

$$
\rho(r)=\rho_{0} r_{0}^{2} /\left(r_{0}^{2}+r^{2}\right)
$$

the radial to the vortex axis Coulomb field $E_{r}$ is:

$$
r E_{r}=2 \pi \rho_{0} r_{0}^{2} \ln \left(\left(r_{0}^{2}+r^{2}\right) / r_{0}^{2}\right)
$$

For the particle drift velocity (Fig. 2):

$$
V_{\varphi}=c E_{r} / H
$$

at large distances from the axis, we have a movement with constant circulation (with logarithmic precision) and solid-type rotation on the small ones. The logarithmic factor reflects the slow decrease of the charge density at large distances.

The quasi-classical quantization is possible by using the condition:

$$
m r V_{\varphi}=n \cdot \hbar
$$

which leads to the angular frequency of the bunch:

$$
\Omega_{n}=n \cdot \hbar / m r^{2}
$$

\footnotetext{
${ }^{8}$ However, sporadic GP can occur at all phases [45]
} 


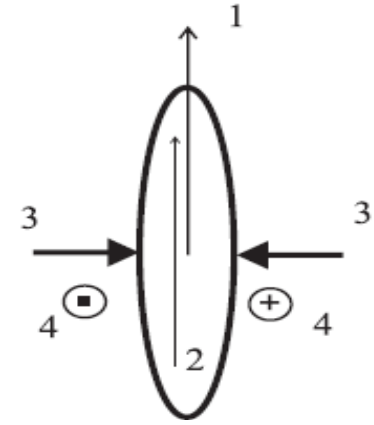

Fig. 2 In the discharge bunch filament the Coulomb particle repulsion leads to rotation in the crossed electric and magnetic fields around the bunch axis. This rotation transforms the bunch into an electromagnetic tornado. For electrons and positrons the rotation directions are opposite. The rotation can cause the circular polarization which is observed in GPs. 1: Magnetic field; 2: bunch velocity; 3: radial electric field of space charge; 4 : drift velocities.

The particle linear density $\mathrm{N}=\pi r_{0}^{2} \cdot \rho_{0} / e$ and, correspondingly, the (linear density) current of the vortex tube $c e N$ are quantized. As a result, they do not contain any model parameters and depends on the magnetic field only:

$$
c e N=n \cdot \hbar B / m
$$

here, $n$ is integer or half-integer. The full Goldreigh-Julian current flowing through the gap is provided by $q$ vortex lines as:

$$
q=\Omega m \Sigma_{P C} / 2 \hbar n \ln \left(R / r_{0}\right)^{2}
$$

(the logarithmic factor is being determined by the quadratic density decrease). In the ground state, it is required for that $q \approx 10^{7}$ lines. For a smaller $q$, the Rydberg states with $n \gg 1$ is required. Discharges may arise while the charges flow down from the microscopic edges of the polar cap surface, forming bunches and low frequency radiation that feeds the cavity. The edges may be destroyed in this process with later restoration. Rotation frequencies form the bands the borders of which are determined by tornado internal and external radii. For example, the radius $r \approx$ $10^{-5} \mathrm{~cm}$ corresponds to the frequency $\Omega \approx 10^{10} \mathrm{~s}^{-1}$. Such structure might explain the frequency bands [34] observed in GP spectrum ${ }^{9}$.

\footnotetext{
${ }^{9}$ These strips have been observed in the interpulse spectrum only. According to Ref. [18], the strips correspond to Bernstine modes.
}

\subsection{Power-Law Distributions}

In the scheme under consideration, the energy emanated in the GP is proportional to the break area. The observed power-law of GPs occurrence frequency in accordance with their energy [35] means the power dependence of a break appearance probability upon the break area. Here the analogy with the geophysical phenomena in which many examples of such distributions are known seems to be quite relevant. Many observed statistical patterns causing power-low distributions can be obtained under the elementary assumption of the small correlation time of random force with different physical definition of "forces" and "particles" [36]. The consequence of such a force correlation is the dependence of the form $N(\geq E) \propto E^{-1}$ for the cumulative frequency of the events with "energy" $E$ which explains many empirical dependencies [36]. Here, apparently, it is necessary to add frequency of the GP pulsar appearance where the energy is proportional to the break areas in the magnetosphere. With smaller energies $E$, the GP complies with the power-law [36] $N(\geq E) \propto E^{-a}(a=0.9-1.1)$. With greater energies, a sharp bend in the distribution is observed. It can easily be interpreted as the result of the break superposition which leads to the steepening of the distribution $(a>2)$.

\section{Conclusions}

Now, we summarize the foregoing. In the giant pulses, the energy density is comparable with that of oscillation in the inner gap. It means that we obtain radiation at the GP instants directly from the gap by-passing the magnetosphere plasma. It becomes possible if the breaks or the cracks and holes appear in magnetosphere due to random allocation of plasma-generating discharges. GP duration is not always fully attributable to its physical nature. The pulse length may be determined by the dynamics of the break appearance and disappearance in the 
magnetosphere. The GP power-law intensity distribution is also determined by the probability of appearing the breaks of different size and is not directly associated with GP radiation physics. The appearance of breaks is most probable in the phases that conform to the "waveguides" near the magnetic axis or near the edge of open field lines. Separate discharges may be superimposed on the radiation through the breaks. In such cases, the relativistic aberration [49] narrows down the angle range $\Delta \varphi$ up to $10^{-7}$ and fast rotation contracts the pulse duration $\Delta t$ $=P \Delta \varphi / 2 \pi$ up to $10^{-9} \mathrm{~s}$. Obviously, circular polarization of GP is also governed by physics of its radiation. Such a polarization can be generated by electromagnetic tornados in the gap.

To sum up, a simple analogy with the Sun radiation is observed on a cloudy day. In the breaks in the clouds, we observe some kind of a "pulse". A large break lets more energy get through (an analogue of the GP intensity) but it is the characteristic of the break and not of the emitter. The "pulse" duration is determined by the break existence but not by the physics of the radiation. The steepness of the "pulse" is also determined by the movement of the clouds but not by the solar processes. The emitter's main characteristic is the brightness temperature and spectrum as well as polarization. But fast fluctuations such as solar flares and bursts of solar radiation could make their own contribution if we can see them between the clouds.

\section{Acknowledgements}

I am grateful to Usov, V. V. for support of the idea of this work, to Beskin, V. S. and Istomin, Ya. N. for calling my attention to the paper [10], and to Ul'yanov O. M. for helpful discussion and comments on the text.

\section{References}

[1] Manchester, R. N. 2009. Neutron Stars and Pulsars. Berlin, Heidelberg: Springer-Verlag, 33-5.

[2] Kuzmin, A. D. 2007. "Giant Pulses of Pulsar Radio
Amission.” Astrophys. \& Space Sci. 308: 563-7.

[3] Knight, H. S. 2006. "Observational Characteristics of Giant Pulses and Related Phenomena." Chin. J. Astron. Astrophys. 6 (2): 41-7.

[4] Soglasnov, V. A. 2006. "Amazing Properties of Giant Pulses and the Nature of Pulsar's Radio Emission." In Proceedings of the 363 WE-Heraeus Seminar on: "Neutron Stars and Pulsars", MPE Report 291, 68-71.

[5] Cairns, I. H. 2004. "Properties and Interpretations of Giant Micropulses and Giant Pulses from Pulsars." Astrophys. J. 610: 948-55.

[6] Staelin, D. H., and Sutton, J. M. 1970. "Observed Shape of Crab Nebula Radio Pulses.” Nature 226: 69-71.

[7] Hankins, T. H., and Eilek, J. A. 2007. "Radio Emission Signatures in the Crab Pulsar.” Astrophys. J. 670: 693.

[8] Hankins, T. H., Kern, J. S., Weatherall, J. C., and Eilek, J. A. 2003. "Nanosecond Radio Bursts from Strong Plasma Turbulence in the Crab Pulsar." Nature 422: 141-3.

[9] Popov, M. V., and Stappers, B. 2007. "Statistical Properties of Giant Pulses from the Crab Pulsar." Astron. \& Astrophys. 470: 1003-7.

[10] Soglasnov, V. A., Popov, M. V., Bartel, N., Cannon, W., Novikov, A. Y., and Kondratiev, V. I. 2004. "Giant Pulses from PSR B1937+21 with Widths 15 Nanoseconds and $\mathrm{T}_{b} \geq 5 \times 10^{39} \mathrm{~K}$, the Highest Brightness Temperature Observed in the Universe." Astrophys. J. 616: 439-51.

[11] Kuiper, L., Hermsen, W., and Stappers, B. 2004. "Chandra and RXTE Studies of the X-ray/Gamma-ray Millisecond Pulsar PSR J0218+4232." Adv. Space Res. 33: 507-12.

[12] Cusumano, G., Hermsen, W., Kramer, M., Kuiper, L., Lohmer, O., Massaro, E., Mineo, T., Nicastro, L., and Stappers, B. W. 2003. "The Phase of the Radio and X-ray Pulses of PSR B1937+21.” Astron. \& Astrophys. 410: 9-12.

[13] Ulyanov, O. M., Zakharenko, V. V., and Deshpande, A. 2007. "Two-Frequency Observation of Six Pulsars Using UTR-2 and GEETEE Radio Telescopes." Radio Physics and Radio Astronomy 12: 5-19.

[14] Usov, V. V. 1987. "On Two-Stream Instability in Pulsar Magnetosphere.” Astrophys. J. 320: 333-5.

[15] Asseo, E., Pelletier G., and Sol, H. 1990. "A Non-linear Radio Pulsar Emission Mechanism.” Mon. Not. R. Astr. Soc. 247: 529-48.

[16] Weatherall, J. C. 1997. "Modulational Instability, Mode Conversion, and Radio Emission in the Magnetized Pair Plasma of Pulsars." Astrophys. J. 483: 402-13.

[17] Istomin, Y. 2003. "Origin of Giant Radio Pulses." In Proceedings of IAU Symp. 218, Young Neutron Stars \& Their Environments, 62-4.

[18] Lyutikov, M. 2007. "On Generation of Crab Giant Pulses." 
Mon. Not. R. Astr. Soc. 381: 1190.

[19] Petrova, S. A. 2004. "On the Origin of Giant Pulses in Radio Pulsars.” Astron. \& Astrophys. 424: 224-36.

[20] Ruderman, M. A., and Sutherland, P. G. 1975. "Theory of Pulsars: Polar Cap, Sparks, and Coherent Microwave Radiation.” Astrophys. J. 196: 51-72.

[21] Sturrock, P. A. 1971. “A Model of Pulsars.” Astrophys. J. 164: 529-56.

[22] Young, M. D. T. 2004. "A Resonant-Mode Model of Pulsar Radio Emission." In Proceedings of IAU Symp. 218, Young Neutron Stars and Their Environments, 365.

[23] Kontorovich, V. M. 2007. "Dice and Pulsars." Problems of Atomic Science and Technology 3 (1): 195-9.

[24] Bliokh, P. V., Nikolaenko, A. P., and Filippov, Y. F. 1977. Global Electro Magnetic Resonances in the Cavity Earth-Ionosphere. Kiev: Naukova Dumka, 200.

[25] Kontorovich, V. M., and Flanchik, A. B. 2008. "On the Connection between Gamma and Radio Radiation Spectra in Pulsars." JETP 106: 869-77.

[26] Arons, J., and Scharleman, E. T. 1979. "Pair Formation above Pulsar Polar Caps: Structure of the Low Altitude Acceleration Zone.” Astrophys. J. 231: 854-79.

[27] Beskin, V. S., Gurevich, A. G., and Istomin, Y. N. 1993. Physics of the Pulsar Magnetosphere. Cambridge: Cambridge University Press.

[28] Beskin, V. S. 2010. MHD Flows in Compact Astrophysical Objects. Germany: Springer-Verlag, 425.

[29] Kontorovich, V. M. 2008. "On the Nature of High Brighteness Temperatures of the Pulsar Giant Pulses." In Proceedings of Conference on Physics of Neutron Stars, Russia.

[30] Muslimov, A. G., and Harding, A. K. 2004. "High-Altitude Particle Acceleration and Radiation in Pulsar Slot Gaps." Astrophys. J. 606: 1143-53.

[31] Ershov, A. A., and Kuzmin, A. D. 2003 "Detection of Giant Pulses the Pulsar B1112+50." Astron. Lett. 29: 91-5.

[32] Kondratiev, V. I., Popov, M. V., Soglasnov, V. A., Kovalev, Y. Y., Bartel, N., and Ghigo, F. 2006. "Detailed Study of Giant Pulses from the Millisecond Pulsar B1937+21." In Proceedings of the 363. WE-Heraeus Seminar on: "Neutron Stars and Pulsars", 76-9.

[33] Knight, H. S., Bailes, M., Manchester, R. N., and Ord, S. M. 2005. "A Search for Giant Pulses from Millisecond Pulsars.” Astrophys. J. 625: 951-6.

[34] Eilek, J. A., and Hankins, T. H. 2007. "What Makes the Crab Pulsar Shine?" In Proceedings of Confernce on "Forty Years of Pulsars: Millisecond Pulsars, Magnetars and More", 51-5.

[35] Bilous, A. V., Kondratiev, V. I., Popov, M. V., and Soglasnov, V. A. 2007. "Review of Overall Parameters of Giant Radio Pulses from the Crab Pulsar and B1937+21."
In Proceedings of Conference on "Forty Years of Pulsars: Millisecond Pulsars, Magnetars and More”, 118-20.

[36] G.S. Golitsyn, Statistics and Dynamics of Natural Processes and Phenomena: Methods, Tools and Results. Moscow: KRASAND (2013) (in Russian).

[37] Romani, R. W., and Jonston, S. 2001. "Giant Pulses from the Millisecond Pulsar.” Astrophys .J. Lett. 557: 93-6.

[38] Jonston, S., and Romani, R. W. 2003. "Giant Pulses from PSR B0540-69 in the Large Magellanic Cloud." Astrophys. J. Lett. 590: 95-8.

[39] Joshi, B. C., Kramer, M., Lyne, A. G., McLaughlin, M., and Stairs, I. H. 2004. "Giant Pulses in Millisecond Pulsars." In Proceedings of IAU Symp. on Young Neutron Stars and Their Environments, 319-20.

[40] Kuzmin, A. D., Ershov, A. A., and Lozovsky, B. Y. 2004. "Detection of Giant Pulses from the Pulsar B0031-07." Astron. Lett. 30: 247-50.

[41] Ershov, A. A., and Kuzmin, A. D. 2005. "Detection of Giant Pulses in Pulsar PSR J1752+2359." A\&A 433: 593.

[42] Kuzmin, A. D., and Ershov, A. A. 2006. "Detection of Giant Radio Pulses from the Pulsar B0656+14.” Astron. Lett. 32: 650 .

[43] Luo, Q., and Melrose, D. 2008. "Oscillating Pulsar Polar Gaps.” Mon. Not. R. Astron. Soc. 387: 1291.

[44] Popov, M., Kuzmin, A. D., Ulyanov, O. M., Deshpande, A. A., Ershov, A. A., and Zakharenko, V. V. 2006. "Instantaneous Radio Spectra of Giant Pulses from the Crab Pulsar from Decimeter to Decameter Wavelengths." Astron. Rep 83: 630-7.

[45] Slowikowska, A., Jessner, A., Kanbach, G., and Klein, B. 2006. "Comparison of Giant Radio Pulses in Young Pulsars and Millisecond Pulsars." In Proceedings of the 363 WE-Heraeus Seminar on: Neutron Stars and Pulsars (Posters and contributed talks) Physikzentrum Bad Honnef, 64.

[46] Jessner, A., Slowikowska, A., Klein, B., Lesch, H., Jaroschek, C. H., and Kanbach, G. 2005. "Giant Radio Pulses from the Crab Pulsar." Adv. Space Res. 35: 1166-71.

[47] Bhat, R. N. D., Tingay, S. J., and Knight, H. S. 2008. "Bright Giant Pulses from the Crab Nebula Pulsar: Statistical Properties, Pulse Broadening and Scattering Due to the Nebula." Astrophysical Journal 676: 1200-9.

[48] Popov, M. V., Soglasnov, V. A., Kondratiev, V. I., Bilous, A. V., Moshkina, O., and Oreshko, V. V. 2009. "Multifrequency Study of Giant Radio Pulses from the Crab Pulsar with the K5 VLBI Recording Terminal." Astron. Soc. Japan 61: 1197-209.

[49] Gil, J., and Melikidze, G. I. 2005. "Angular Beaming and Giant Subpulses in the Crab Pulsar." Astron. \& Astrophys. 432: 61-5.

[50] Anderson, P. W. 1958. "Absence of Diffusion in Certain Random Lattices.” Phys. Rev. 109: 1492-505. 
[51] Gredeskul, S. A., and Freilikher, V. D. 1990. "Localization and Wave Propagation in Randomly-Layered Media." Soviet Phys. Uspekhi 160: 239-62.

[52] Papanicolaou, G. C. 1971. "Wave Propagation in a One-Dimensional Random Medium." SIAM, J. Appl. Math. 21: 13-8.

[53] Klyatskin, V. I. 1980. Stochastic Equations and Waves in Stochastic Heterogeneous Mediums. Moscow: Nauka.

[54] Lifshits, I. M., Gredeskul, S. A., and Pastur, L. A. 1982. Introduction to the Theory of Disordered Systems. Moscow: Nauka, 359. (in Russian)

[55] Volokitin, A. S., Krasnosel'skikh, V. V., and Machabeli,
G. Z. 1985. "Waves in the Relativistic Electron-Positron Plasma of a Pulsar." Sov. J. Plasma Phys. 11: 310-38.

[56] Arons, J., and Barnard, J. J. 1986. "Wave Propagation in Pulsar Magnetospheres: Dispersion Relations and Normal Modes of Plasmas in Superstrong Magnetic Fields." Astrophys. J. 302: 120-37.

[57] Lominadze, J. G., Machabeli, G. Z., and Melikidze, G. I. 1986. "Pulsar Magnetosphere Plasma." Sov. J. Plasma Phys. 12: 1233.

[58] Kontorovich, V. M. 2010. "Quantized Electromagnetic Tornado in Pulsar Vacuum Gap.” JETP 110: 966-72.

[59] Davidson, R. C. 1974. Theory of Non Neutral Plasmas. London: W. A. Benjamin, Inc.

\section{Appendix A: On Nontransparency of the Pulsar Magnetosphere in the Open Field Lines Region}

Electrodynamics of the boundary of the polar gap with plasma in the region of open field lines, which limits the gap from above, is a fairly challenging task and has not been sufficiently developed, although it was announced in the work [22]. Therefore, we give below the following physical considerations indicating that this boundary should have relatively high reflective properties, so that the gap could be considered as a good resonator. Now we would like to focus our attention primarily on the random environment (the electron-positron plasma) which has to pass through the wave from the inner gap before leaving it.

This randomness is the result of random character of the discharges on the surface of the polar cap [23]. Discharges generate the plasma via the birth of electron-positron pairs in a magnetic field (by gamma-ray photons emitted at breakdowns). The geometry of the open field lines and the strong magnetic field create a configuration that is close to the one-dimensional one, in which the predominant role is played by the backward scattering that results in Anderson localization [50]. With such scattering in a stationary random environment the reflected wave travels the path along which all the randomphase shifts acquired by the wave along the straight path are fully compensated for. This leads to an exponentially small average transmission coefficient of the wave through a layer of random medium exceeding the localization length [51] $l$ and, accordingly, to an exponentially small difference between the average reflection coefficient $R$ and unity [52, 53]:

$$
\bar{R} \approx 1-\frac{\pi^{5 / 2}}{2}\left(\frac{L}{l}\right)^{-3 / 2} \exp \left(-\frac{L}{4 l}\right)
$$

where, $L$ is the thickness of the layer. (The wave transmission occurs only on the most favorable paths that are different for different random realizations [54]; they are reported in the text in a simplified manner as the breaks in the magnetosphere). In order to render the use of these findings reliable it is necessary to make sure that the situation is actually quite close to one-dimensional and that the nonstationarity of configurations (in the unchallenged stationary random process) does not destroy the desired coherence of scattering. (Nonstationarity may occur even on average [43]). This question is left open now and we hope to return to this issue separately.

This does not contradict the fact that in a smoothly inhomogeneous magnetized relativistic electron-positron plasma with identical distributions of electrons and positrons the transverse electromagnetic waves have the gapless nature [55-57] and, seemingly, can easily penetrate through the magnetosphere ${ }^{10}$. However, the magnetosphere is not the smoothly inhomogeneous, especially in the area of open field lines, and this is a basic fact for the problem of reflection of electromagnetic waves. But besides this, in the transition layer between the gap and the plasma the electromagnetic waves is of a different character. Indeed, there is a strong longitudinal electric field in the transition layer, which turns the positrons, that are born in pairs, back to the star.

Therefore, there should be a layer of non-relativistic positron plasma with the Goldreich-Julian plasma frequency

${ }^{10}$ The most frequent objection to the resonance properties of the inner gap. Let us note, that the waves are linearly. 
$\omega_{G J}=\sqrt{\omega_{c} \Omega} \approx 10^{10} s^{-1}$, (here $\omega_{c}$ is the cyclotron frequency and $\Omega$ is the angular frequency of star rotation). Note that the upper edge of the pulsar radio spectrum, as remarked in [25], is located close to this frequency. It is important, however, to highlight one more circumstance. The back scattering is strongly suppressed in the relativistic outflowing plasma. But in the transition layer in which the reverse flow of the positrons is accelerated up to the speed of light, the backscattering of the waves is also very efficient due to the same relativistic aberration. This should lead to the opacity of the plasma and to the high reflective properties of the upper lid of the gap resonator. It will be recalled that the existence of the cavity is one of assumptions in this paper. We analyze the consequences of this assumption, which in fact allows us to give a consistent explanation of all the facts relating to the issue of GPs.

\section{Appendix B: Energy Density Estimation}

The average energy density $U$ of the low-frequency electromagnetic field in the gap can be estimated from the energy conservation law in the cavity excited by external discharge currents. The conservation law averaged over time yields the balance condition div $S$ $=-j_{e x} E \sim$, where, $S$ is the Poynting vector, $j_{e x}$ is the density of the spark currents and $E \sim$ is the strength of the low-frequency electric field. The "brackets denotes" averaging over time. By the average values of $\left\langle A>\right.$ we imply their mean square values $\sqrt{<A^{2}>}$. Integrating the balance equation over the volume of the resonator and using the Gauss' theorem we obtain $\oint<S>d \Sigma=$ $-\int \mathrm{dV}<\mathrm{j}_{\mathrm{ex}} \mathrm{E}_{\sim}>$. The average value $\int \mathrm{d} V<j_{e x} E_{\sim}>$ is estimated with Cauchy-Bunyakovsky inequality $\int \mathrm{d} V<j_{e x} E_{\sim}>$ $\leq \int \mathrm{d} V<j_{e x}><E_{\sim}>$. For the average current density $\left\langle j_{e x}>\right.$ we take the Goldreich-Julian value $<j_{e x}>=c \rho_{G J}$ where the Goldreich-Julian charge density is $\rho_{G J}=-\Omega B /(2 \pi c)$. Using the theorem about the average for the integral in the right-hand side of the balance condition inequality we obtain the estimate $\int\left\langle\mathrm{j}_{\mathrm{ex}} \mathrm{E}_{\sim}\right\rangle d V \leq \mathrm{c} \rho_{\mathrm{GJ}}\langle E\rangle \Sigma_{\mathrm{PC}} \mathrm{h}$. Here $h$ is the height of the gap, $\Sigma_{P C}$ is the area of the polar cap. Note that the power emitted in the radio-frequency region is $\left.I_{R}=\oint<S\right\rangle d \Sigma=c U_{R} \Sigma_{W}$, where $U_{R}=U /(1+$ $\mu), U=\left\langle E_{\sim}^{2}>/ 4 \pi\right.$ and the contribution of the closed modes is $u \approx\left(\omega_{1} / \omega_{m n}\right)_{R}^{\alpha-1}\left[1-\left(\omega_{m n} / \omega_{1}\right)_{R}^{\alpha-1}\right]$. Inserting these relations into the balance condition we have $(1+\mu)_{c}^{-1} \frac{\left\langle E_{\sim}^{2}\right\rangle}{4 \pi} \Sigma_{w} \leq c \rho_{G J}<E_{\sim}>\Sigma_{P C}$ and whence we estimate the average electric field in the gap: $<E_{\sim}>\leq 4 \pi(1+\mu) \rho_{G J} h \Sigma_{\mathrm{PC}} / \Sigma_{w}$. Finally, raising to the square we find the upper bound for the energy density in the gap (1): $\mathrm{U}=$ $<E_{\sim}^{2}>/(4 \pi) \leq 4 \pi(1+\mu)^{2}\left(\Sigma_{P C} / \Sigma_{w}\right)^{2}\left(h \rho_{G J}\right)$, whence follows under the typical values of parameters that:

$$
U \leq 10^{16}(1+\mu)^{2} \operatorname{erg} / \mathrm{cm}^{3} .
$$

On the other hand, we can make an independent estimate of $U$ by expressing the average field $\left\langle E_{\sim}>\right.$ through the intensity of radio emission $I_{R}$. Using the balance condition in the form $\oint\langle S\rangle d \Sigma=c \rho_{\mathrm{GJ}} \cdot\left\langle E_{\sim}\right\rangle \cdot \Sigma_{P C} h$ we have estimate $\left\langle E_{\sim}>\right.$ limited from below $I_{R} \leq c \rho_{\mathrm{GJ}} \Sigma_{P C} h\left\langle E_{\sim}\right\rangle$ which leads to the lower bound for the energy density of oscillations in the gap Eq. (2):

$$
\frac{1}{4 \pi}\left(\frac{I_{R}}{c \rho_{G J} \Sigma_{P C} h}\right)^{2} \leq \mathrm{U}
$$

\section{Appendix C: Electromagnetic Tornado}

Let us consider an electromagnetic tornado in the gap [58]. The equations of motion in the plane orthogonal to a magnetic field in complex variables $\xi=x+i y$ take the form $\mathrm{d} w / \mathrm{d} t-i \omega_{c} w=(e E / m r) \xi, w=\mathrm{d} \xi / \mathrm{d} t$, where $\mathbf{E}=E \cdot \mathbf{r} / r, \mathbf{r}=(x, y)$, is the field of a space charge, and $\omega_{c}=e H / m c$ is the cyclotron frequency. In the axial electric field $E=E(\mathrm{r})$ depending solely on the distance $r$ from the axis, which we believe to be a constant parameter, these equations allow solutions of the type $\xi=\xi_{0}(\mathrm{r}) e^{i \Omega(r) t}$. The frequencies $\Omega$ obey the equation $\Omega^{2}-\omega_{c} \Omega+e E / m r=0$, roots of which are equal to $2 \Omega_{ \pm}=\omega_{\mathrm{c}} \pm \sqrt{\omega_{\mathrm{c}}^{2}-4 e E / m r}$. Transition to the 1-system in which the bunch moves with relativistic velocity results in replacing $\Omega \rightarrow \Omega / \Gamma$ where $\Gamma$ is the Lorentz-factor. In this derivation, the appearance of the azimuthal magnetic field in the 1-system, which is induced by the longitudinal electric current should be taken into account. This field can be found from the Maxwell equations. In the pulsar conditions there is a small parameter 
$4 e E / m r \omega_{c}^{2} \ll 1$ the expansion by which yields the values $\Omega_{ \pm} \approx \omega_{\mathrm{c}}$ and $\Omega_{-} \approx \mathrm{cE} / \mathrm{rH}$. The first root is in agreement with the common cyclotron mode slightly modified by the electric field. The second root, being of basic interest to us, corresponds to the drift in the crossed fields. We may check it by going over to the polar coordinates through substitution $\xi=r e^{i} \varphi$. The equations for the radial and azimuthal velocities $V_{r}=\mathrm{d} r / \mathrm{d} t, V_{\varphi}=r \mathrm{~d} \varphi / \mathrm{d} t \quad$ take the forms $\frac{\mathrm{d} V_{r}}{\mathrm{~d} t}-\frac{V_{r}^{2}}{r}+\omega_{c} V_{\varphi}-\frac{e E}{m}=0$, $\frac{\mathrm{d} V_{\varphi}}{\mathrm{d} t}=-V_{r}\left(\frac{V_{\varphi}}{r}-\omega_{c}\right)$. The stationary solution, in which the azimuthal velocity is time-independent, has to satisfy the conditions $\frac{V_{\varphi}^{2}}{r}-\omega_{c} V_{\varphi}+\frac{e E}{m}=0,\left(\frac{V_{\varphi}}{r}-\omega_{c}\right) \cdot V_{r}=0$. They are held at $V_{r}=0$ and $V_{\varphi}=r \cdot \Omega_{ \pm}$, where $\Omega_{ \pm}$are the frequencies found above and having the meaning of angular velocities of rotation (cf. [59]). The solution $V_{\varphi}=r \Omega_{-}$describes the electromagnetic tornado in the polar gap in which the repulsion field of the space charge is compensated by the Lorentz force, the radial movement is absent, and rotation is specified by the drift in the crossed fields. Due to this rotation, the circular polarization appears in the discharge radiation.

\section{Appendix D Intermediate Epilogue}

Of a report 2008: [A1] arXiv:0911.3272, on high brightness temperature of pulsar giant pulses.

Later we have found that in the inner polar gap of pulsar should generate strong (coherent) electromagnetic oscillations during acceleration of electrons in an increasing (from zero at the surface of the star [26]) longitudinal (with respect to the magnetic field) electric field [A2]. When this field is large enough (narrow internal gap), these oscillations win the competition and at sufficiently low frequencies are the dominant mechanism of em waves generation [A3]. Electron acceleration in the increased from zero at the star surface a longitudinal electric field passes through a maximum and tends to zero as the electron velocity approaches to the speed of light. Therefore, at high frequencies, this mechanism of radiation weakens [A2, A3] and gives place to other mechanisms of radiation (relativistic, beam-plasma, magnetic resonance, etc., see the references in the main text and also, for example, in [A2]). The emission spectrum when averaged over the polar cap becomes a power law with a high-frequency (hf) breakage [A2]. This is the physical cause of the power law spectrum and its break at radiation due to longitudinal acceleration in the gap.

This conclusion is consistent with the Pushchino sample of the Malofeev's catalog [A4] of pulsar spectra with hf break depending on the period as the square root of the inverse period of the pulsar [A5]. In the theory of radiation at longitudinal acceleration [A2], this corresponds to the frequency dependence of the hf break as the root of the ratio of the magnetic field to the period ${ }^{11}$ [A2, A3]. In turn, in the theory of the accelerating electric field [A6, 28, A7], this dependence corresponds to a narrow gap, i.e. strong accelerating field. The Pushchino sample itself contains enough powerful pulsars [A8]. The last were specially selected by Malofeevn, V. M. in compiling the catalog for the possibility to trace the features of the spectrum at frequencies far from the peak of the radiation, i.e., while the power is substantially reduced. In favor of the fact that the emission of pulsar of the Pushchino sample at low frequencies is due to longitudinal acceleration says also a correlation between the hf break and the frequency of maximum of the spectrum [A5], which provides a theoretical basis in [A3]. On the other hand, for the Crab pulsar there is an opportunity to explain the differences in the spectra of the main pulse and interpulse [7, 8, 34, A9]: when changing the mechanisms of radiation in the gap with increasing frequency, the relativistic mechanism with narrow beam focused, by virtue of the geometry of the pulsar magnetic field, in the direction of the line of sight of interpulse [A10] comes into play. It is possible that the emission occurs in narrow jets [28, A7], as

\footnotetext{
${ }^{11}$ This fact is used by us in [A19, A20] to determine the magnitude of the magnetic field of single pulsars. Assuming the magnetic dipole losses thus also possible to determine the angle between the magnetic axis and the axis of rotation.
} 
expected in his time in the model of Ruderman and Sutherland [20] with a strong accelerating field at the surface of the star. However, unlike in this model, here the jets appear due to peculiar regime of acceleration in the electric field increasing from zero [A3]: the advantage in the acceleration have sections with higher temperature due to (thermal) fluctuations and, consequently, with the greater value of initial electron velocity. Current flows further warms this area, and thus must occur instability of uniform flow, expressed in the formation of jets. Such jets are rotating in crossed fields-magnetic field of the pulsar and electric field of the space charge of the same stream, forming an electromagnetic "tornado" [A1, A11, 58]. This helps to explain the emergence of circular polarization of giant pulses, which can reach $100 \%$ [8]. In favor the existence of such "tornado" tells us the appearance of bands in the spectra of inter pulse [7] (for the Crab pulsar)-while in the spectra of the main pulse the bands are not observed. Explanation requires attraction (semiclassical) quantization of rotation in the tornadoes [A12, A13]. Evidence of the existence of strong oscillations in the gap can also serve a correlation between gamma radiation and giant pulses [A14]. Such a correlation component, apparently, is in the gamma-ray emission of the Crab pulsar [A15]. Gamma radiation in this case arises as a result of the inverse Compton scattering of accelerated relativistic electrons at the low-frequency oscillations in the gap [25, A16]. The presence of powerful low frequency oscillations of the radio band in the inner gap of the pulsar is reflected in the processes of particle acceleration and the birth of gamma rays in the electric field of the gap, and, consequently, on the birth electron-positron plasma in the magnetosphere. The energy density oscillations in the gap becomes an important additional parameter on which depend the equilibrium conditions in the pulsar. In the work [A17] have shown that the condition of the birth of electron-positron pairs, taking into account the losses on Compton scattering of low-frequency photons in the gap, limits on top the power of low frequency oscillations. Has been found the condition that the Compton losses on scattering of low-frequency photons are responsible for the shutdown of pulsars. Have been obtained estimates of energy density oscillations in the gap on the plane "derivative period-period". These estimates are consistent with the results obtained by the power and spectrum of the observed radio and gamma radiation of pulsars [A17]. Thus appeared a number of additional in relation to discussed in our report [A1] data confirming the presence of powerful oscillations in the inner gap. For their existence is not necessarily the presence of trapped modes. Other arguments of this paper [A1] remain valid. New details and discussion see also in [A18]. In the perspective-the explanation of the phase shift of inter pulse radiation from its window that is observed on the "middle" radio frequencies in the Crab pulsar [A9]. This mysterious effect is naturally explained by reflection from the surface of the star the radiation of relativistic particles with a narrow diagram, flying across the gap to the surface of the pulsar. It can be as positron emission flying to the star from the magnetosphere as well as electrons on the "non-preferred" field lines [A7]. Reflected from the surface of the star that radiation is directed at the mirror angle to the magnetic field line on which the particles fly, and consequently, to the direction of the radiation cone particles flying from the surface and form the radiation in the window of interpulse. Absence of displacement of interpulse position at lower and higher frequencies [A9] is easily attributed to the change of the prevailing mechanism of radiation (in this case-the relativistic emission in the inner polar gap). Furthermore, it seems quite realistic in this model the solution of the problem of coherence emission in relativistic and non-relativistic mechanism, which we hope to present on court of readers in the near future.

\section{The References to Epilogue}

[A1] Kontorovich, V. M. 2008. "On High Brightness Temperature of Pulsar Giant Pulses.” In the 8th International Conference on Physics of Neutron Stars, Saint-Petersburg.

[A2] Kontorovich, V. M., and Flanchik, A.B. 2013. "High Frequency Cut-off and Changing of Radio Emission Mechanism in Pulsars.” Astrophys.Space Sci. 345: 169.

[A3] Kontorovich, V. M., and Flanchik, A. B. 2013. "Correlation of Pulsar Radio Emission Spectrum with Peculiarities of Particle Acceleration in a Polar Gap.” JETP 116 (1): 80-6.

[A4] Malofeev, V. M. 1999. Catalog radio Spectra of Pulsars. Pushchino: PRAO ASC FIAN. (in Russian) 
[A5] Malov, I. F. 2004. Radio Pulsars. Moscow: Nauka. (in Russian)

[A6] Dyks, J., and Rudak, B. 2000. “Approximate Expressions for Polar Gap Electric Field of Pulsars.” Astron. \& Astrophys. 362: 1004.

[A7] Harding, A. K. 2013. “The Neutron Star Zoo.” Frontiers of Physics 8 (6): 679.

[A8] Malofeev, V. M. 1996. "Pulsar Radio Spectra". In Pulsars: Problems \& Progress ASP Confer. Series, 271.

[A9] Moffett, D., and Hankins, T. 1996. “Multifrequency Radio Observations of the Crab Pulsar.” Astrophys. J. $468: 779$.

[A10] Kontorovich, V. M., and Flanchik, A. B. 2011. "Physics of Neutron Stars." In Proceedings of 2011 International Conference on Physics of Neutron Stars, 75.

[A11] Kontorovich, V. M. 2010. "Giant Pulses of Pulsars.” Problems of Atomic Science and Technology 4 (68): 143-8. (in Russian)

[A12] Kontorovich, V. M. 2014. "Electromagnetic Tornado Semiclassical Quantization and Origin of the Bands in the Giant Pulses Frequency Spectrum of the Crab Pulsar.” Presented at 2014 International Conference on Physics of Neutron Stars, Russia.

[A13] Kontorovich, V. M. 2014. "The Quantization of the Electromagnetic Tornado and Origin of Bands in the Spectrum of Giant Pulses of the Crab Pulsar." Astronomy Letters 40 (12): 793-9.

[A14] Flanchik, A. B., and Kontorovich, V. M. 2012. "Gamma Radiation of Pulsars as Result of Inverse Compton Scattering at Acceleration of Electrons in a Pulsar Polar Gap.” Problems of Atomic Science and Technology 77: 125-9.

[A15] Bilous, A. V., Kondratiev, V. I., McLaughlin, M. A., Mickaliger, M., Ransom, S. M., and Lyutikov, M. 2011. "Correlation of Fermi Photons with Highfrequency Radio Giant Pulses from the Crab Pulsar." Astrophys. J. 728:110-9.

[A16] Kontorovich, V. M., and Flanchik, A. B. 2011. "The Theory of Radio Emission in the Inner Gap and Correlation with Gamma Radiation in Pulsars." In "High Energy Astrophysics Today and Tomorrow”, Moscow.

[A17] Kontorovich, V. M., and Flanchik, A. B. 2010. "The Influence of Powerful Low-Frequency Oscillations in the Vacuum Gap on the Acceleration of Electrons and the Shutdown Line in Pulsars." Problems of Atomic Science and Technology 4 (68): 170-5. (In Russian)

[A18] Palfreyman, J. L., Hotan, A. W., Dickey, J. M., Young, T. G., and Hotan, C. E. 2011. "Consecutive Bright Pulses in the Vela Pulsar.” Astrophys J.L. 735: 17-9.

[A19] Kontorovich, V. M. 2012. “Analogs of the Earth-Ionosphere Cavity in Theories of Pulsar Radio Emission.” In Proceedings of Conf. EMES 2012, Kharkov. http://ri.kharkov.ua//emes/EMES2012_Thesis.pdf.

[A20] Kontorovich, V. M. 2013. “The Magnetic Fields of Radio Pulsars.” Astronomy Reports 59 (4): 277-87. 